\title{
Autoimmune Pancreatitis Associated with Idiopathic Retroperitoneal Fibrosis: A Case Report
}

\author{
Takayuki Aimoto ${ }^{1}$ Eiji Uchida ${ }^{1}$, Yoshiharu Nakamura ${ }^{1}$, Akira Katsuno, \\ Kazumitsu Chou ${ }^{1}$, Takashi Tajiri ${ }^{1}$ and Zenya Naito ${ }^{2}$ \\ ${ }^{1}$ Surgery for Organ Function and Biological Regulation, Nippon Medical School Graduate School of Medicine \\ ${ }^{2}$ Second Department of Pathology, Nippon Medical School
}

\begin{abstract}
A 69-year-old man presented with obstructive jaundice and dark urine. Contrast-enhanced computed tomography revealed an enlarged pancreas with homogenous enhancement. Endoscopic retrograde pancreatography demonstrated short-segmental, irregular narrowing of the main pancreatic duct. The patient underwent exploratory laparotomy and needle biopsies of the pancreas, which showed marked fibrotic change with lymphocyte infiltration. These clinicopathologic findings suggested autoimmune pancreatitis. Four years later, computed tomography demonstrated marked periaortic soft tissue surrounding a calcified infrarenal abdominal aorta compatible with retroperitoneal fibrosis. We diagnosed retroperitoneal fibrosis with noncontiguous pancreatic fibrosis. This patient responded well to corticosteroid treatment. Autoimmune pancreatitis associated with idiopathic retroperitoneal fibrosis seems to be extremely rare, and to our knowledge, only a few cases have been reported.

(J Nippon Med Sch 2006; 73: 235-239)
\end{abstract}

Key words: autoimmune pancreatitis, idiopathic retroperitoneal fibrosis, multifocal idiopathic fibrosclerosis, IgG4, steroid therapy

\section{Introduction}

Chronic pancreatitis of autoimmune origin is a relatively new and increasingly reported clinicopathologic entity. Autoimmune pancreatitis (AIP) is characterized by an absence of acute attacks of pancreatitis, hypergammaglobulinemia, irregular narrowing of the main pancreatic duct, sonolucent swelling of the pancreatic parenchyma, lymphoplasmacytic inflammation of the pancreas, and a good response to glucocorticoid treatment ${ }^{1-3}$.
This pancreatic disease is encountered in various other autoimmune diseases, including idiopathic retroperitoneal fibrosis (IRF), sclerosing cholangitis, and Sjögren's syndrome. ${ }^{4}$ To our knowledge, only a few cases of AIP with IRF have been reported ${ }^{56}$. We describe a patient with AIP, in whom retroperitoneal fibrosis developed 4 years later.

\section{Case Report}

A 69-year-old man was admitted to our hospital because of jaundice and dark urine. The patient had

Correspondence to Takayuki Aimoto, MD, Department of Surgery, Nippon Medical School, 1-1-5 Sendagi, Bunkyoku, Tokyo 113-8603, Japan

E-mail: aimoto@maple.ocn.ne.jp

Journal Website (http://www.nms.ac.jp/jnms/) 


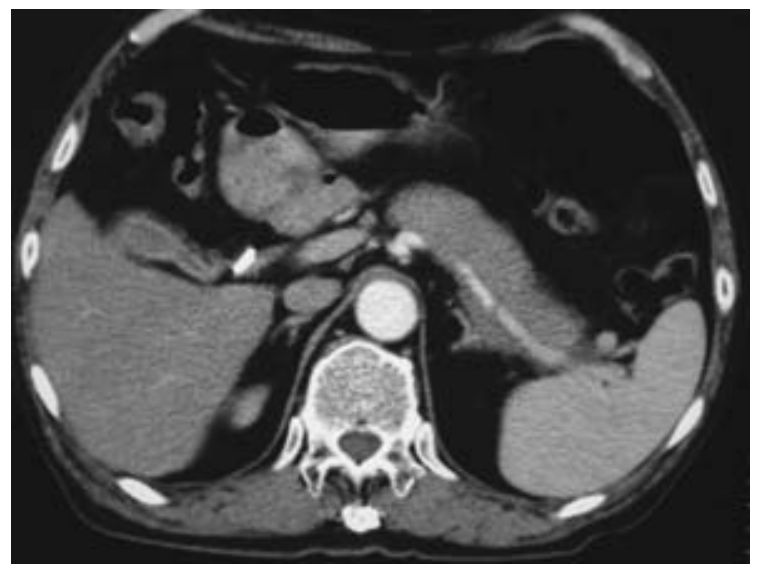

Fig. 1A Contrast-enhanced CT scan revealed an enlarged pancreas with homogenous enhancement.

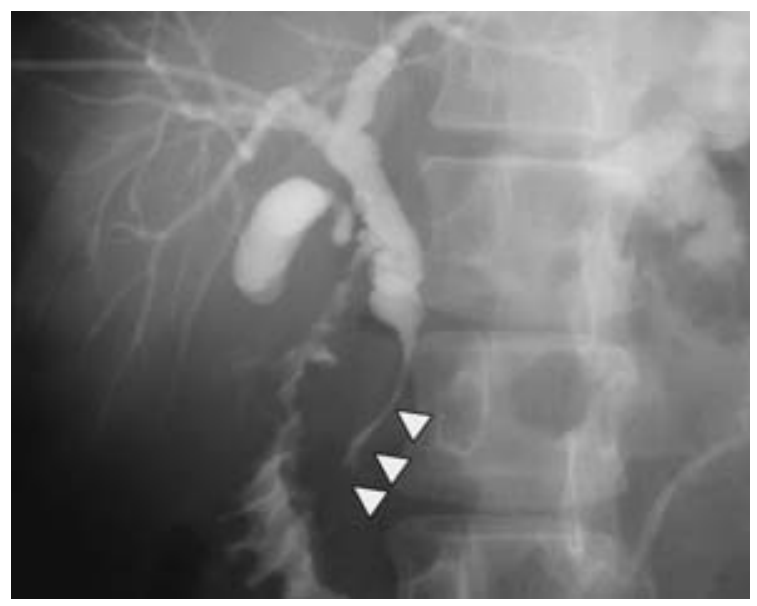

Fig. 1B Percutaneous transhepatic cholangiography showed severe stenosis of the lower common bile duct (white arrowheads).

no history of drug or alcohol use. Physical examination revealed icterus and normal vital signs. Examination indicated that the heart and lungs were normal. The liver and spleen were not palpable, and there was no evidence of an abdominal mass.

Laboratory investigations revealed liver dysfunction (aspartate aminotransferase, $223 \mathrm{IU} / \mathrm{L}$ [normal, 10 $28 \mathrm{IU} / \mathrm{L}$; alanine aminotransferase, 428 IU/L [normal, 5 33 IU/L]; alkaline phosphatase, 732 $\mathrm{IU} / \mathrm{L} \quad$ [normal, $\quad 66 \sim 220 \quad \mathrm{IU} / \mathrm{L}$ ]; $\quad \gamma$-glutamyl transpeptidase, 1,057 IU/L [normal, 8 $59 \mathrm{IU} / \mathrm{L}$; total serum bilirubin, $16.2 \mathrm{mg} / \mathrm{d} l$ [normal, $0.2 \sim 1.2 \mathrm{mg} / \mathrm{d} l$ ]; direct serum bilirubin, $14.5 \mathrm{mg} / \mathrm{d} l$ [normal, $0.1 \sim 0.4$ $\mathrm{mg} / \mathrm{d} l])$. Serological tests for hepatitis, smooth

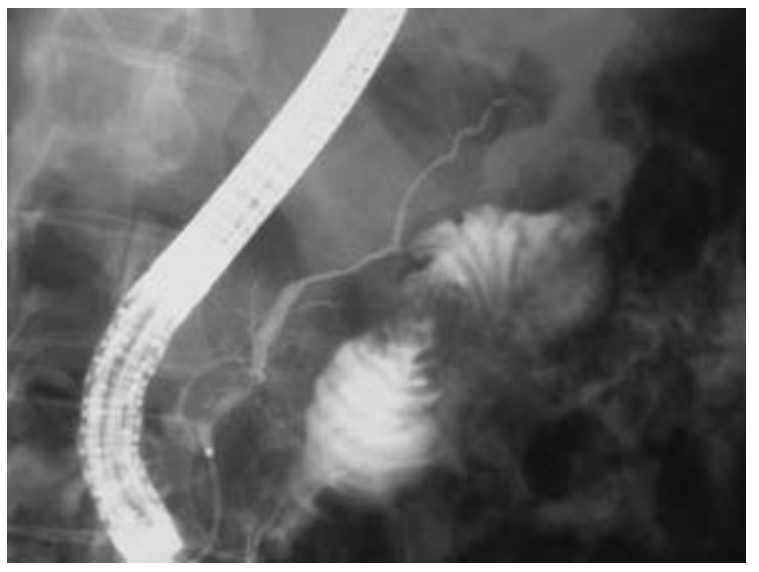

Fig. 1C Endoscopic retrograde pancreatography demonstrated short-segmental, irregular narrowing of the main pancreatic duct.

muscle antibodies, and antimitochondrial antibodies were negative. Antinuclear antibodies were positive with a titer of 1 : 70 (uniform pattern). Serum protein electrophoresis revealed increased levels of immunoglobulin G (IgG) $(2,536 \mathrm{mg} / \mathrm{d} l$; normal, $788 \sim$ $1,841 \mathrm{mg} / \mathrm{d} l)$ and IgG4 $(195 \mathrm{mg} / \mathrm{d} l$; normal, 6 140 $\mathrm{mg} / \mathrm{d} l)$. Tumor marker levels were as follows: carcinoembryonic antigen, $0.8 \mathrm{ng} / \mathrm{d} l$ [normal, $<2.5$ $\mathrm{ng} / \mathrm{d} l$ ]; carbohydrate antigen19-9, $78 \mathrm{U} / \mathrm{ml}$ [normal, < $37 \mathrm{U} / \mathrm{mll}$.

Abdominal ultrasonography showed a diffusely enlarged and hypoechoic pancreas. Contrastenhanced computed tomography $(\mathrm{CT})$ revealed an enlarged pancreas with homogenous enhancement ( Fig . 1 A ) . Percutaneous transhepatic cholangiography showed severe stenosis of the lower common bile duct (Fig. 1B). Endoscopic retrograde pancreatography demonstrated shortsegmental, irregular narrowing of the main pancreatic duct (Fig. 1C). The patient underwent exploratory laparotomy with needle biopsies of the pancreas. Histologic examination of the biopsy specimens showed marked fibrotic change with lymphocyte infiltration but no malignant cells. Because a definitive tissue diagnosis of malignancy was not made and because these clinicopathologic findings suggested AIP, cholecystectomy and choledochojejunostomy were performed. Postoperatively, the patient was treated with ursodeoxycholic acid $(900 \mathrm{mg} / \mathrm{d} l)$ alone.

The patient was followed up as an outpatient. 


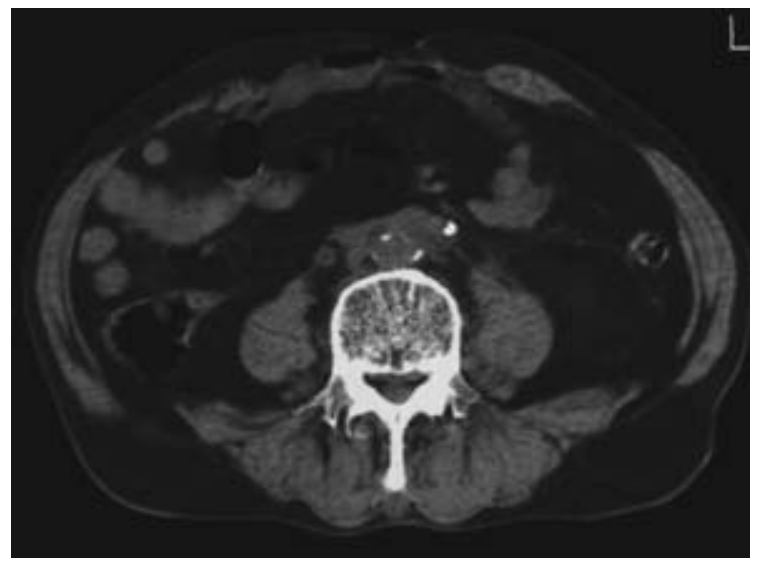

Fig. 2A CT demonstrated marked periaortic soft tissue surrounding a calcified infrarenal abdominal aorta compatible with retroperitoneal fibrosis.

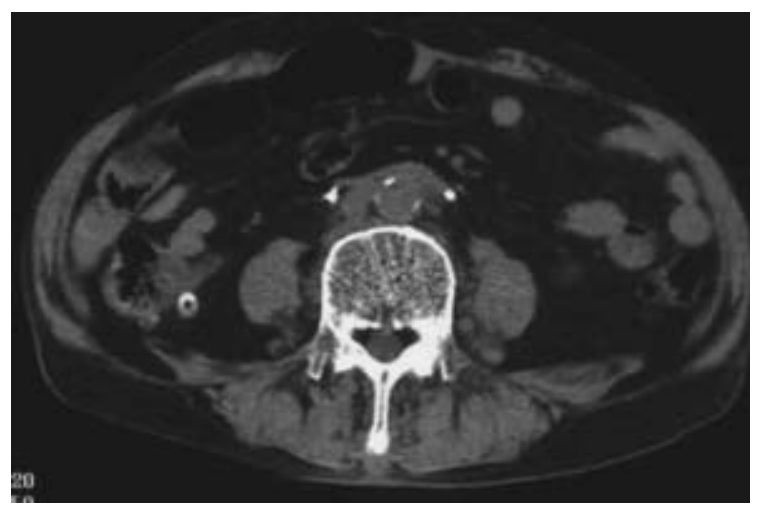

Fig. 2B Follow-up CT scan demonstrated a reduction in size of the periaortic soft tissue.

Four years later, abdominal ultrasonography detected right hydronephrosis and intravenous pyelography revealed a nonfunctioning right kidney. CT demonstrated marked periaortic soft tissue surrounding a calcified infrarenal abdominal aorta compatible with retroperitoneal fibrosis (Fig. 2A). Abdominal CT scan also detected pancreatic stone formation and parenchymal atrophy in the body and tail of the pancreas (Fig. 3). We diagnosed retroperitoneal fibrosis resulting in right hydronephrosis, which was initially managed by ureteral catheterization. Immediately afterwards, we started to treat the patient with $40 \mathrm{mg}$ prednisolone daily for 2 weeks, after which we reduced the dose by $5 \mathrm{mg}$ per week until a daily dose of $5 \mathrm{mg}$ was reached. Six months after the start of corticosteroid

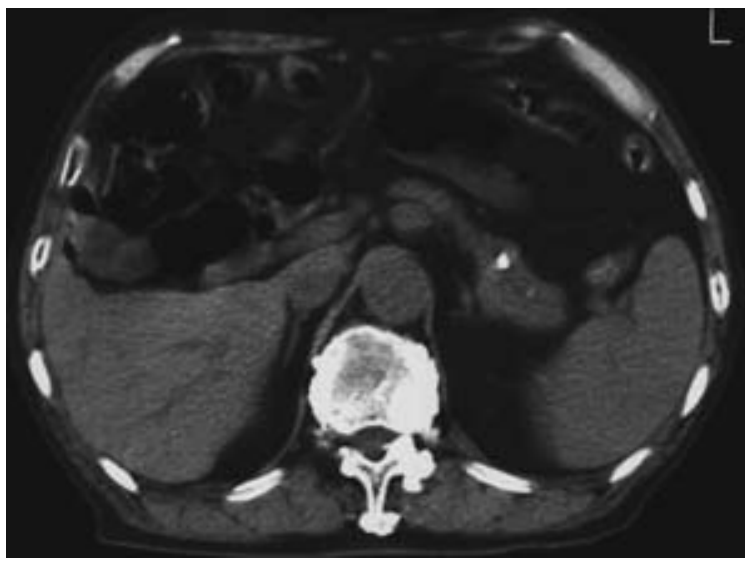

Fig. 3 Abdominal CT scan detected pancreatic stone formation and parenchymal atrophy in the body and tail of the pancreas

therapy, the serum IgG4 concentration had decreased to $55 \mathrm{mg} / \mathrm{d} l$, and follow up CT demonstrated a reduction in the size of periaortic soft tissue (Fig. 2B).

The patient is well and is being followed up as an outpatient.

\section{Discussion}

AIP is a relatively new clinicopathologic entity, variously referred to as sclerosing pancreatitis, primary inflammatory pancreatitis, and nonalcoholic duct-destructive chronic pancreatitis ${ }^{2.3}$. AIP has the following characteristics: it is generally painless, causing much less pain than other types of pancreatitis; it is not alcohol-related; and it shows increased serum $\gamma$-globulin or IgG levels, the presence of autoantibodies, irregular narrowing of the pancreatic duct, an enlarged and elastic-hard pancreas, no cysts, no calcifications, lymphocyte, or plasma cell infiltration with fibrosis, and a good clinical response to steroid therapy ${ }^{2,3}$. Hamano et al. ${ }^{7}$ have found that patients with AIP have elevated serum concentrations of immune complexes and of the IgG4 subclass and that these concentrations decrease significantly during glucocorticoid therapy. These findings suggest that the pathogenesis of this disease is closely related to an autoimmune abnormality. AIP is occasionally associated with other autoimmune diseases, such as primary sclerosing cholangitis, chronic idiopathic 
inflammatory bowel disease, Sjögren's syndrome, systemic lupus erythematosus, and $\mathrm{IRF}^{4}$.

IRF is an uncommon entity characterized by the formation of a dense plaque of fibrous tissue in the retroperitoneal space. The fibrous tissue usually constricts the ureters ${ }^{8}$. The vena cava, the aorta, and mesenteric vessels are rarely involved ${ }^{9}$. Retroperitoneal fibrosis usually spreads diffusely through the retroperitoneal space, not focally, and may show continuous extension into the mesentery or into and around the pancreas or duodenum. Contiguous extension of retroperitoneal fibrosis or sclerosing mesenteritis into the pancreas has been well reported ${ }^{10-12}$.

IRF most commonly presents as an isolated finding, but rarely it can be a feature of multifocal idiopathic fibrosclerosis (MIF). MIF is a rare disease representing proliferation of fibrous tissue at different locations and may be manifested as IRF, pancreatic fibrosis, mesenteric fibrosis, mediastinal fibrosis, pseudotumor of the orbit, Riedel's thyroiditis, pelvic fibrosis, or sclerosing cholangitis ${ }^{13}$. In the setting of MIF, pancreatic fibrosis can present as a pseudotumor with obstructive jaundice, and other features of MIF with pancreatic fibrosis are sclerosing cholangitis and orbit pseudotumor ${ }^{14,15}$.

As the present case was initially diagnosed with AIP, there was no evidence of retroperitoneal fibrosis. Four years later, pelvic CT demonstrated marked periaortic soft tissue that was not continuous with the pancreas but was compatible with retroperitoneal fibrosis. IRF with noncontiguous pancreatic fibrosis is extremely rare: to our knowledge, only 8 cases, including the present case, have been reported ${ }^{5,6,16-18}$. All patients were men older than 50 years. In seven of the eight cases, serum concentrations of $\mathrm{IgG}$ or IgG4 were elevated. Antinuclear antibodies were detected in four cases. Hamano et al. $^{6}$ have shown abundant infiltration of IgG4-bearing plasma cells in both the ureteral and pancreatic tissues and a reduction in serum IgG4 levels after treatment with corticosteroids. These findings show that pancreatic fibrosis is associated with retroperitoneal fibrosis and can be complicated by MIF. Moreover, immunologic findings suggest that an autoimmune process is involved in the pathophysiology of AIP and retroperitoneal fibrosis in these cases. Steroid therapy was effective in six of the eight patients. Mitchinson et al. ${ }^{19}$ have concluded that corticosteroids constitute an important adjunct or an alternative to surgical treatment in patients with IRF alone. This rare entity might show a good response to corticosteroid therapy similar to that of IRF.

On the other hand, alcohol-induced chronic pancreatitis is a progressive disease with recurrent attacks which results in the formation of pancreatic stones, irregular dilatation of the pancreatic duct, and atrophy of the parenchyma with fibrosis. In contrast to alcohol-induced pancreatitis, AIP is characterized by irregular narrowing of the main pancreatic duct, sonolucent swelling of the parenchyma and the absence of pancreatic stone formation, which had been reported to be a characteristic feature of this condition. However, our patient relapsed and had pancreatic stone formation 4 years after the initial presentation. Takayama et $\mathrm{al}^{20}$ have reported that chronic AIP shows severe fibrosis, pancreatic stones, and irregular dilatation of pancreatic ducts, as in ordinary chronic pancreatitis. Therefore, AIP might be a progressive disease with pancreatic lithiasis.

In conclusion, we have reported an unusual case of autoimmune pancreatitis with IRF, which can be complicated by MIF. This rare disorder should be promptly recognized so that corticosteroid therapy can be started immediately.

\section{References}

1. Sarles H, Sarles JC, Muratore R, Guien C: Chronic inflammatory sclerosis of the pancreas-An autonomous pancreatitis disease? Am J Dig Dis 1961; 6: 688-698.

2. Yoshida K, Toki F, Takeuchi T, Watanabe S, Shiratori K, Hayashi N: Chronic pancreatitis caused by an autoimmune abnormality: proposal of the concept of autoimmune pancreatitis. Dig Dis Sci 1995; 40: 1561-1568.

3. Uchida K, Okazaki K, Konishi Y, et al.: Clinical analysis of autoimmune-related pancreatitis. Am J Gastroenterol 2000; 95: 2788-2794.

4. Kamisawa T, Funata N, Hayashi Y, et al.: Close relationship between autoimmune pancreatitis and multifocal fibrosclerosis. Gut 2003; 52: 683-687. 
5. Chutaputti A, Burrell MI, Boyer JL: Pseudotumor of the pancreas associated with retroperitoneal fibrosis: A dramatic response to corticosteroid therapy. Am J Gastroenterol 1995; 90: 1155-1158.

6. Hamano H, Kawa S, Ochi Y, et al.: Hydronephrosis associated with retroperitoneal fibrosis and sclerosing pancreatitis. Lancet 2002; 359: 1403-1404.

7. Hamano H, Kawa S, Horiuchi A, et al.: High serum IgG4 concentrations in patients with sclerosing pancreatitis. N Engl J Med 2001; 344: 732-738.

8. Ormond JK: Bilateral ureteral obstruction due to envelopment and compression by an inflammatory retroperitoneal process. J Urol 1948; 59: 1072-1079.

9. Haynes IG, Simon J, West RJ, Hamer JD: Idiopathic retroperitoneal fibrosis with occlusion of the abdominal aorta treated by transluminal angioplasty. Br J Surg 1982; 69: 432-433.

10. Renner IG, Ponto GC, Savage III WT, Boswell WD: Idiopathic retroperitoneal fibrosis producing common bile duct and pancreatic duct obstruction. Gastroenterol 1980; 79: 348-351.

11. Cappell MS: Obstructive jaundice due to retroperitoneal fibrosis involving the head of the pancreas. J Clin Gastroenterol 1994; 18: 53-56.

12. Udd M, Jarvinen HJ, Haglund $\mathrm{CH}$ : Retroperitoneal fibrosis of the pancreatic head mimicking carcinoma of the pancreas. Eur J Surg 2001; 167: 148-150.

13. Comings DE, Skubi KB, Van Eyes J, Motulsky AG: Familial multifocal fibrosclerosis. findings suggesting that retroperitoneal fibrosis, mediastinal fibrosis, sclerosing cholangitis, Riedel's thyroiditis and pseudotumor of the orbit may be different manifestations of a single disease. Ann Intern Med 1967; 66: 884-892.

14. Clark A, Zeman RK, Choyke PL, et al.: Pancreatic pseudotumors associated with multifocal idiopathic fibrosclerosis. Gastrointest Radiol 1988; 13: 30-32.

15. Levey J, Mathai J: Diffuse pancreatic fibrosis: an uncommon feature of multifocal idiopathic fibrosclerosis. Am J Gastroenterol 1998; 93: 640-642.

16. Wiesner $\mathrm{W}$, Kocher $\mathrm{T}$, Beglinger $\mathrm{C}$, Harder F, Steinbrich W: Pseudotumor of the pancreatic head associated with idiopathic retroperitoneal fibrosis. Dig Surg 2001; 18: 418-421.

17. Fukukura Y, Fujiyoshi F, Nakamura F, Hamada H, Nakajo M: Autoimmune pancreatitis associated with idiopathic retroperitoneal fibrosis. AJR 2003; 181: 993-995.

18. Uchida K, Okazaki K, Asada M, et al.: Case of chronic pancreatitis involving an autoimmune mechanism that extended to retroperitoneal fibrosis. Pancreas 2003; 26: 92-94.

19. Mitchinson MJ, Withycombe JFR, Jones AR: The response of idiopathic retroperitoneal fibrosis to corticosteroids. Br J Urol 1971; 43: 444-449.

20. Takayama M, Hamano H, Ochi Y, et al.: Recurrent attacks of autoimmune pancreatitis result in pancreatic stone formation. Am J Gastroenterol 2004; 99: 932-937.

(Received, April 17, 2006)

(Accepted, May 16, 2006) 